\title{
Level Densities and $\gamma$ Strength Functions in Light Sc and Ti Isotopes
}

\author{
A. Bürger ${ }^{1}$, A.C. Larsen ${ }^{1}$, N.U.H. Syed ${ }^{1}$, M. Guttormsen ${ }^{1}$, S. Harissopulos ${ }^{2}$, M. Kmiecik ${ }^{3}$, T. Konstantinopoulos ${ }^{2}$, \\ M. Krtička ${ }^{4}$, A. Lagoyannis ${ }^{2}$, T. Lönnroth ${ }^{5}$, K. Mazurek ${ }^{3}$, M. Norby ${ }^{5}$, H. Nyhus ${ }^{1}$, G. Perdikakis ${ }^{2}$, S. Siem ${ }^{1}$, A. Spyrou $^{2}$, \\ and A. Voinov 6 \\ 1 University of Oslo, Norway \\ 2 NCSR "Demokritos", Athens, Greece \\ 3 IFJ PAN, Krakow, Poland \\ 4 Charles University, Prague, Czech Republic \\ 5 Åbo Akademi, Åbo, Finland \\ 6 Ohio University, Athens, OH, USA
}

\begin{abstract}
We present preliminary results from a measurement of nuclear level densities and the $\gamma$-ray strength
\end{abstract} of light $\mathrm{Sc}$ and $\mathrm{Ti}$ isotopes using the Oslo Method.

\section{Introduction}

Nuclear level densities and $\gamma$-ray strength functions are important ingredients in calculations of stellar isotope abundances, but also in the prediction of nuclear reactor burnup products. Therefore, a continuing effort has since long been devoted to the study of these nuclear properties both experimentally and theoretically.

The nuclear physics group at the University of Oslo has in the last years concentrated on the study of these nuclear properties using a procedure often called the "Oslo Method". In this work, we present preliminary results obtained using this method for light $\mathrm{Sc}$ and Ti isotopes, among them the first application of the Oslo Method to an isotope produced in a $(p, \alpha)$ reaction.

In the next section, the experimental setup is described, followed in section 3 by the preliminary results obtained so far. In the final section 4 , an outlook will be given.

\section{Experimental Setup}

The experiment was performed at the cyclotron laboratory of the University of Oslo. A proton beam with an energy of $32 \mathrm{MeV}$ impinged on a ${ }^{46} \mathrm{Ti}$ target of $3 \mathrm{mg} / \mathrm{cm}^{2}$ thickness enriched to $86 \%{ }^{46} \mathrm{Ti}$. Eight individually collimated silicon $\Delta E-E$ particle detectors were placed in forward direction after the target at an angle of $45^{\circ}$ with respect to the beam axis. The target was surrounded by the $\gamma$-ray detector array CACTUS consisting of 28 collimated NaI scintillator crystals.

Using the specific energy losses in the thin $(140 \mu \mathrm{m})$ $\Delta E$ and the thick $(1500 \mu \mathrm{m}) E$ Si counters, $p, d, t$, and $\alpha$ ejectiles were identified to select specific reaction channels. From the known $Q$-values, the reaction kinematics and the energy losses in the materials passed through by the particles, the initial excitation energies $E_{x}$ of the produced nuclei could be reconstructed. The excited nuclei will emit cascades of $\gamma$ rays to decay to their ground states. The energy spectra of these $\gamma$ rays were measured in coincidence with the particles, and matrices $E_{x}$ vs. $E_{\gamma}$ was constructed after correcting for the $\mathrm{NaI}$ response function as described in [1]. The first-generation method [2] was then applied on this matrix to extract a matrix $P$ containing the spectrum of primary $\gamma$-ray energies $E_{\gamma}$ for each initial excitation energy $E_{x}$.

From the matrix $P$, the shape of both the level density and the shape of the $\gamma$-ray strength function can be extracted. To determine absolute values for the level density and the strength function, data from other experiments have to be utilized. The procedure is described in detail in [1] and the articles referenced therein.

\section{Results}

At low energies, all the level densities presented in the following are normalised to the density of discrete known levels extracted from the ENSDF database [3] for the respective isotope.

\subsection{Results for ${ }^{43} \mathrm{Sc}$}

At energies about $2 \mathrm{MeV}$ above the proton separation energy, the level density has been normalised to proton resonance spacings extracted from the RIPL database [4]. Unfortunately, many of the resonances lack a definitive spin assignment. To perform the normalisation, it has therefore been assumed that all tentative assignments are correct, and that the distribution of unknown spin-parity values equals 


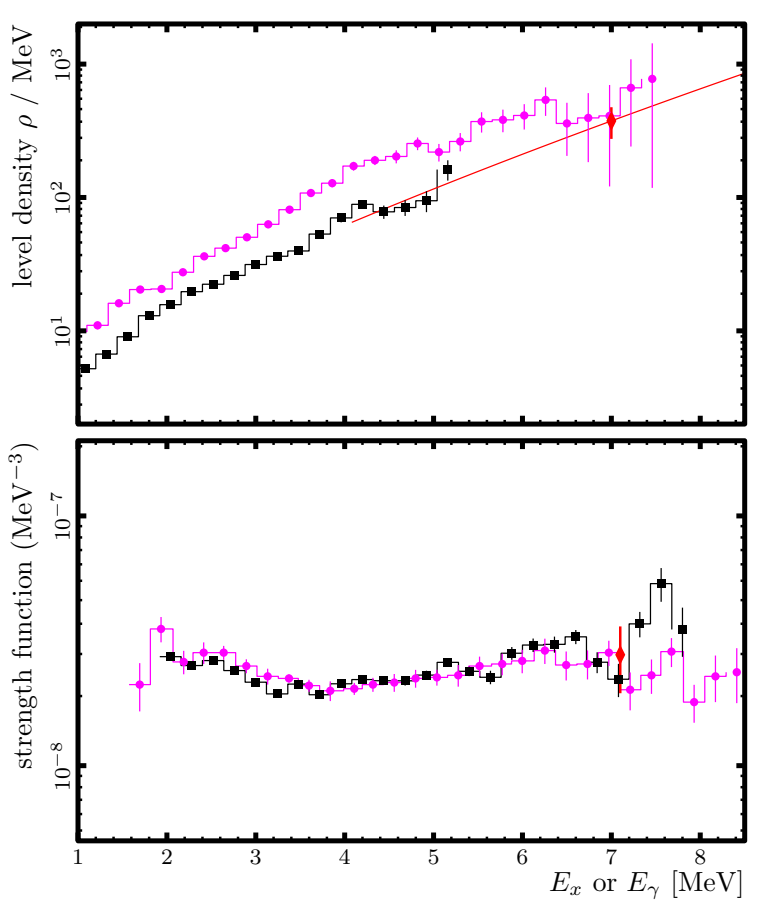

Fig. 1. Preliminary nuclear level density as function of the excitation energy $E_{x}$ (upper panel) and $\gamma$-ray strength function as function of the $\gamma$-ray energy $E_{\gamma}$ (lower panel) for ${ }^{43} \mathrm{Sc}$ (black). The level density and strength function for ${ }^{45} \mathrm{Sc}$ [5] (pink) are shown for comparison. The normalisation data point is shown as a red dot, and the BSFG-model extrapolation of the level density as a red line.

the distribution of known spin values. For the extrapolation to the energy range accessible in this experiment, the back-shifted Fermi-gas model (BSFG) was used. Figure 1 shows the preliminary level density distribution for ${ }^{43} \mathrm{Sc}$. It is apparent that the level density is lower by a factor 2-3 compared to the $A+2$ neighbour ${ }^{45} \mathrm{Sc}$ [5]. While at higher energies this could be understood to be due to the larger number of particles outside the ${ }^{40} \mathrm{Ca}$ core, it is not yet clear how to explain the observed effect for lower energies.

To obtain the $\gamma$-ray strength, a third normalisation point is necessary - usually the average width of neutron resonances is used. As such data are not available for ${ }^{43} \mathrm{Sc}$, the normalisation has been done using the average neutron resonance width of ${ }^{46} \mathrm{Sc}$ (cf. [5]). Figure 1 also shows the preliminary $\gamma$-ray strength function for ${ }^{43} \mathrm{Sc}$. For comparison, the strength function for ${ }^{45} \mathrm{Sc}$ [5] is reproduced in the same figure. Although the two curves have been obtained in very different reactions $-(p, \alpha)$ and $\left({ }^{3} \mathrm{He},{ }^{3} \mathrm{He}\right)-$ the obtained curves are very similar. This is expected for two nuclei so close together in mass, and it indicates that there is no substantial reaction dependence in the data extracted using the Oslo Method. Both curves show an enhancement in the photon strength function for energies below $3 \mathrm{MeV}$. This behaviour has until now been observed in several nuclei, but its origin is not yet understood.

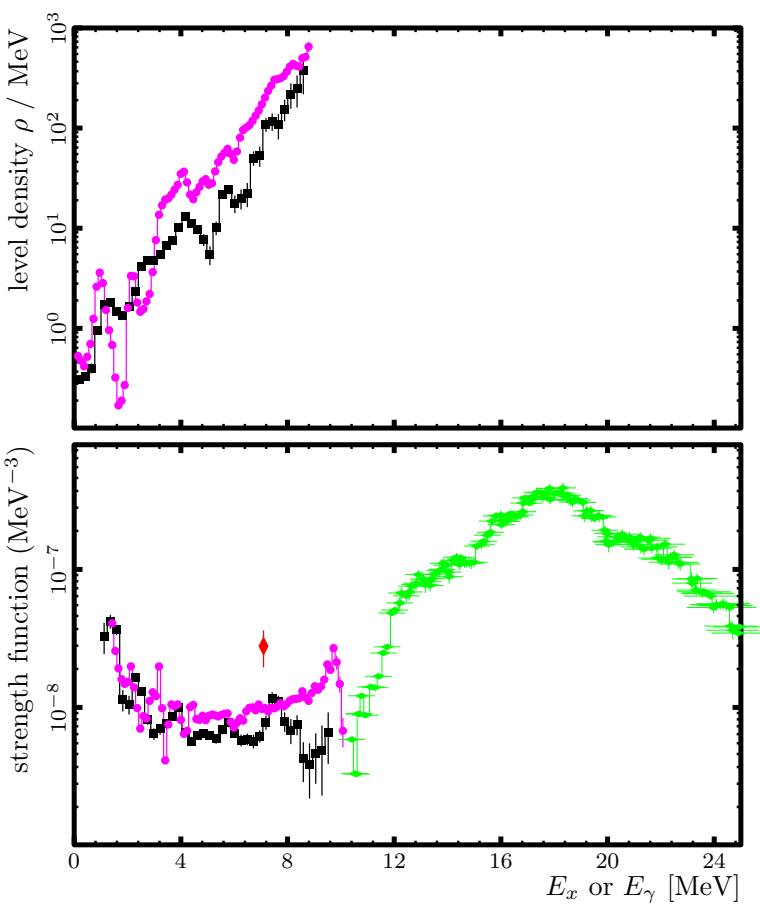

Fig. 2. Preliminary nuclear level densities (upper panel) as function of the excitation energy $E_{x}$ and $\gamma$-ray strength functions as function of the $\gamma$-ray energy $E_{\gamma}$ (lower panel) for ${ }^{44} \mathrm{Ti}$ (black) and ${ }^{46} \mathrm{Ti}$ (pink). The normalisation to external data still has to be performed, only the shape of the functions has been extracted so far. In the lower panel, the photon strength function measured for ${ }^{50} \mathrm{Ti}[6]$ is included to show that a good agreement with the curves obtained in this experiment could be expected.

\subsection{Results for ${ }^{44,46} \mathrm{Ti}$}

For the nuclei ${ }^{44,46} \mathrm{Ti}$, normalisation points have not yet been obtained. The level density and the strength functions for ${ }^{44,46} \mathrm{Ti}$ are presented in fig. 2 . With the preliminary normalisations used in fig. 2, a good agreement with the known level density of ${ }^{50} \mathrm{Ti}$ [6] could be expected. As for the case of ${ }^{43} \mathrm{Sc}$ and ${ }^{45} \mathrm{Sc}$, the level density of the heavier isotope is higher by an almost constant factor - but here, this behaviour is seen only for energies above $3 \mathrm{MeV}$.

\subsection{Results for ${ }^{45} \mathrm{Ti}$}

Regarding the results obtained for ${ }^{45} \mathrm{Ti}$, the reader is referred to the very recent publication [7] where the results of the analysis for this reaction channel are presented and discussed in great detail.

\section{Outlook}

Recently, the experimental setup at the Oslo Cyclotron Laboratory has been upgraded with new Si detectors and electronics.

As shown in fig. 3, the eight new SiRi (Silicon Ring) detectors are each segmented in eight strips of $2^{\circ}$ in $\theta$. 


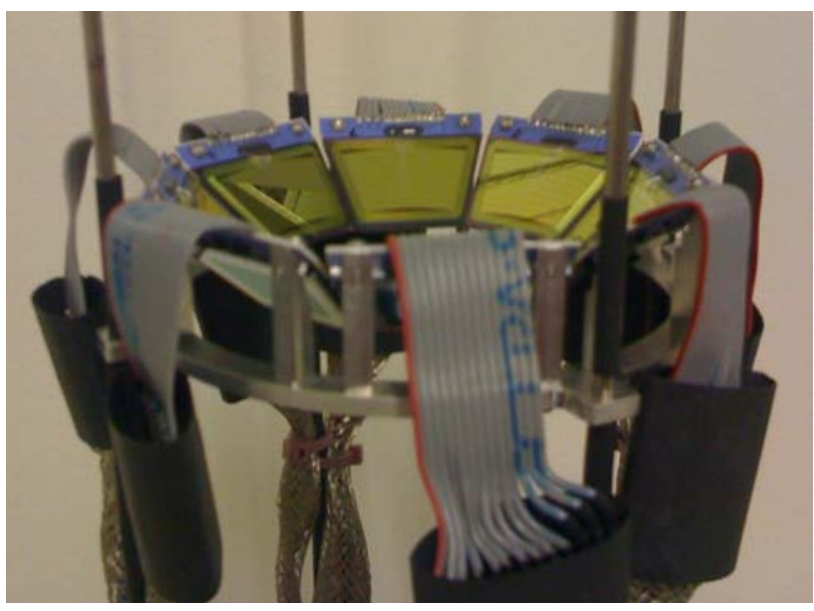

Fig. 3. The new SiRi detectors ready to be mounted in the CACTUS target chamber.

Thus they have a sensitive area enlarged by about a factor 10 compared to the old Si telescopes (depending on the collimator size used with the previous detectors).

A first experiment using the new detectors has been run on ${ }^{90,92} \mathrm{Zr}$ targets using proton beams of 17 and $28 \mathrm{MeV}$, respectively. The data are presently under analysis.

\section{Acknowledgements}

A.B. acknowledges the kind support for his participation in the $\mathrm{CNR} * 09$ conference. The authors wish to thank $\mathrm{E}$. A. Olsen and J. Wikne for excellent experimental conditions. Financial support from the Norwegian Research Council (NFR) is gratefully acknowledged.

\section{References}

1. A. Schiller, L. Bergholt, M. Guttormsen, E. Melby, J. Rekstad, S. Siem, Nucl. Instr. Meth. A 447, 498 (2000), arXiv: nucl-ex/9910009

2. M. Guttormsen, T. Ramsøy, J. Rekstad, Nucl. Instr. Meth. A 255, 518 (1987)

3. ENSDF, Ensdf database (2007), http://www.nndc.bnl.gov/ensdf/

4. RIPL, Ripl database http://www. iaea.org/ripl/

5. A.C. Larsen, M. Guttormsen, R. Chankova, F. Ingebretsen, T. Lönnroth, S. Messelt, J. Rekstad, A. Schiller, S. Siem, N.U.H. Syed et al., Phys. Rev. C 76(4), 044303 (2007), arXiv:0706.0533

6. X.Y. Pywell et al., Nucl. Phys. A 325, 116 (1979)

7. N.U.H. Syed, A.C. Larsen, A. Bürger, M. Guttormsen, S. Harissopulos, M. Kmiecik, T. Konstantinopoulos, M. Krtička, A. Lagoyannis, T. Lönnroth et al., Phys. Rev. C 80(4), 044309 ( 9) (2009), http://link.aps.org/abstract/PRC/v80/e044309 